\title{
Flows of Weights of Crossed Products of Type III Factors by Discrete Groups
}

\author{
By \\ Yoshihiro SEKINE*
}

\section{$\S 0$. Introduction}

In [17] C. Sutherland and M. Takesaki classified (up to cocycle conjugacy) actions of discrete amenable groups on injective factors of type $\mathrm{III}_{\lambda}, 0 \leqq \lambda<1$. For the unique injective factor of type $\mathrm{III}_{1}([7])$, similar classification was recently completed for discrete abelian groups ([14]). This classification is based on four invariants: a certain normal subgroup, the module, the characteristic invariant, and the modular invariant. Cocycle conjugate actions give rise to isomorphic crossed products, and the isomorphism class of an injective type III factor is known to be determined by its flow of weights. Therefore the flow of weights of the crossed product $M \rtimes_{\alpha} G$ should be uniquely determined by the four invariants of the action $\alpha$.

The purpose of this article is to compute the flow of weights of $M \rtimes_{\alpha} G$ explicitly in the case where $M$ is a type III factor and $G$ is a discrete group. This means that we have to compute the center of the crossed product $M \rtimes_{\alpha} G$ by $\mathbb{R}$ relative to a modular automorphism group. Generally a continuous crossed product is difficult to handle because a general element does not admit a Fourier expansion. This difficulty will be avoided by using the canonical extension $\tilde{\alpha}$ of $\alpha$ in the sense of [8] (see Proposition 2.1).

\section{Acknowledgement}

The author would like to express his sincere gratitude to Professor Hideki Kosaki for his helpful suggestions and constant encouragement, and to Professor Toshihiro Hamachi and Professor Motosige Osikawa for their constant encouragement. At last, his most sincere gratitude goes to Professor Jun Tomiyama for his constant encouragement.

Communicated by H. Araki, December 9, 1989.

* Department of Mathematics, Faculty of Science, Tokyo Metropolitan University, Fukazawa, Setagaya-ku, Tokyo 158, Japan. 


\section{$\S 1$. Preliminaries and Main Result}

In this section, we recall some fundamental results which will be used in the sequel, and state the main result.

\subsection{Crossed Products}

Let $M$ be a von Neumann algebra acting on a Hilbert space $H$, and $\alpha: G \rightarrow$ Aut $M$ be an action of a locally compact group $G$ on $M$. Then the crossed product $M \rtimes_{\alpha} G$ is the von Neumann algebra acting on the Hilbert space $L^{2}(G, H)$ generated by $\pi_{a}(x), x \in M$, and $\lambda(g), g \in G$. Here $\pi_{\alpha}(x), \lambda(g)$, are the operators on $L^{2}(G, H)$ defined by

$$
\begin{aligned}
& \left(\pi_{\alpha}(x) \xi\right)(h)=\alpha_{h-1}(x) \xi(h) \\
& (\lambda(g) \xi)(h)=\xi\left(g^{-1} h\right)
\end{aligned} \quad, \quad \xi \in L^{2}(G, H), \quad h \in G,
$$

and satisfy the covariance relation:

$$
\lambda(g) \pi_{a}(x) \lambda(g) *=\pi_{a}\left(\alpha_{s}(x)\right), \quad x \in M, \quad g \in G .
$$

When $G$ is abelian, the dual action $\hat{\alpha}: \hat{G} \rightarrow \operatorname{Aut}\left(M \rtimes_{a} G\right)$ is canonically defined by

$$
\hat{\alpha}_{p}=\operatorname{Ad} v(p), \quad p \in \hat{G} .
$$

Here $\hat{G}$ is the dual group of $G$ and $v(p), p \in \hat{G}$, is the unitary operator on $L^{2}(G, H)$ defined by

$$
(v(\not) \xi)(g)=\overline{\langle g, p\rangle \xi}(g), \quad \xi \in L^{2}(G, H), \quad g \in G .
$$

Then the automorphism $\hat{\alpha}_{p}, p \in \hat{G}$, is characterized by the following:

$$
\begin{aligned}
& \hat{\alpha}_{p}\left(\pi_{\alpha}(x)\right)=\pi_{a}(x), \quad x \in M, \\
& \hat{\alpha}_{p}(\lambda(g))=\overline{\langle g, p\rangle} \lambda(g), \quad g \in G .
\end{aligned}
$$

By Takesaki duality, the second crossed product $\left(M \rtimes_{\alpha} G\right) \rtimes_{\hat{\alpha}} \hat{G}$ is isomorphic to the tensor product $M \otimes B\left(L^{2}(G)\right)$ and the bidual action $\hat{\hat{c}}$ on $\left(M \rtimes_{\alpha} G\right) \rtimes_{\hat{\alpha}} \hat{G}$ corresponds to the action $\alpha \otimes \operatorname{Ad} \rho$ under the above isomorphism, where $\rho$ is the (right) regular representation of $G$ on $L^{2}(G)$. Furthermore, the generators $\pi_{\hat{a}}\left(\pi_{\alpha}(x)\right), \quad x \in M, \pi_{\hat{\alpha}}(\lambda(g)), g \in G$, and $\lambda(p), p \in \hat{G}$, in $\left(M \rtimes_{\alpha} G\right) \rtimes_{\hat{\alpha}} \hat{G}$ are mapped to $\pi_{a}(x), 1 \otimes \lambda_{g}, 1 \otimes v_{p}$, in $M \otimes B\left(L^{2}(G)\right)$ respectively. For details, see [4], [12], or $[18]$.

\subsection{Dual Weights}

Let $M$ be a von Neumann algebra, and let $\alpha: G \rightarrow$ Aut $M$ be an action of a locally compact group $G$ on $M$. Then, for each faithful normal semi-finite weight $\phi$ on $M$, there exists a faithful normal semi-finite weight $\tilde{\phi}$ on $M \rtimes_{\alpha} G$, 
which is called the dual weight of $\phi$. The modular automorphism group of the dual weight $\tilde{\phi}$ is given by

$$
\begin{aligned}
& \sigma_{t}^{\tilde{\phi}}\left(\pi_{\alpha}(x)\right)=\pi_{\alpha}\left(\sigma_{t}^{\phi}(x)\right), \quad x \in M, \\
& \sigma_{t}^{\tilde{\phi}}(\lambda(g))=\Delta_{G}(g)^{i t} \lambda(g) \pi_{\alpha}\left(\left(D \phi \cdot \alpha_{g}: D \phi\right)_{t}\right), \quad g \in G,
\end{aligned}
$$

where $\Delta_{G}$ is the modular function on $G$ and $\left(D \phi \cdot \alpha_{g}: D \phi\right)_{t}$ means Connes' RadonNikodym cocycle $([1])$.

When $(M, H, J, P)$ is a standard form in the sense of [5], the modular operator $\Delta_{\tilde{\phi}}$, the modular conjugation $\tilde{J}$ on $L^{2}(G, H)$ are calculated as follows:

$$
\begin{aligned}
& \left(\Delta_{\tilde{\phi}}{ }^{t} \xi\right)(g)=\Delta_{G}(g)^{i t} \Delta_{\dot{\phi} \cdot \alpha_{g} \cdot \dot{\phi}^{i t} \xi(g)} \\
& (\tilde{J} \xi)(g)=\Delta_{G}(g)^{-1 / 2} u_{g^{-1}} J \xi\left(g^{-1}\right)
\end{aligned}
$$

where $\left\{u_{g}\right\}_{g \in G}$ is the canonical implementation of $\alpha$ ([5]). And, in general, for faithful normal semi-finite weights $\phi, \phi$ on $M$, the relative modular operator $\Delta_{\phi, \psi}$ is calculated by

$$
\Delta_{\dot{\varphi}, \psi^{i t}}=(D \phi: D \phi)_{t} \Delta_{\dot{\varphi}}{ }^{2 t}, \quad t \in \boldsymbol{R} .
$$

For details, see [6].

\subsection{Flows of Weights}

Let $M$ be a type III factor with separable predual. Then the flow of weights of $M$ is realized by a pair of the continuous crossed product $M \rtimes_{\sigma} \phi \boldsymbol{R}$ by the modular action for some faithful normal semi-finite weight $\phi$ on $M$ and the dual action $\theta$ of $\sigma^{\dot{\phi}}$. More precisely, the center $Z$ of $M \rtimes_{\sigma} \dot{R} \boldsymbol{R}$ is isomorphic to $L^{\infty}(X)$ for some standard Borel space $Y$, and by restriction, $\theta$ gives rise to a non-singular ergodic flow $\left\{T_{t}\right\}_{t \in R}$ on $X$ such that

$$
\left(\theta_{t}(f)\right)(x)=f\left(T_{-t} x\right), \quad f \in Z \cong L^{\infty}(X), \quad x \in X .
$$

Thanks to Connes' result on Radon-Nikodym cocycles, the isomorphism class of $M \rtimes_{\sigma} \phi \boldsymbol{R}$ is independent of the choice of a weight $\phi$. Therefore $M \rtimes_{\sigma} \phi \mathbb{R}$ is canonically determined by $M$ and so is the above flow. On the other hand, if $\phi$ is a dominant weight on $M$, there exist a continuous action $\theta: \boldsymbol{R} \rightarrow$ Aut $M_{\dot{\phi}}$ of $\boldsymbol{R}$ on the centralizer of $\phi$ and a faithful normal semi-finite trace $\tau$ on $M_{\dot{\phi}}$ such that

$$
\begin{aligned}
& M \cong M_{\dot{\phi}} \rtimes_{\theta} \boldsymbol{R} \text { (continuous decomposition), } \\
& \tau \cdot \theta_{s}=e^{-s} \tau, \quad s \in \boldsymbol{R}, \\
& \sigma_{t}^{\phi}(\lambda(s))=e^{-i t s} \lambda(s), \quad t, s \in \boldsymbol{R} \text { (under the above identification). }
\end{aligned}
$$

Similarly, the pair $\left(M_{\phi}, \theta\right)$ gives rise to a non-singular ergodic flow. By Takesaki 
duality, however, these flows are conjugate. For an injective type III factor with separable predual, the flow of weights is a complete invariant ([2], [7], [15]). For details, see [3], [18].

\subsection{Invariants for Actions}

Let $\alpha: G \rightarrow$ Aut $M$ be an action of a discrete group $G$ on a type III factor $M$, and let $\left(P_{M},\left\{F_{t}^{M}\right\}_{t \in R}\right)$ be the flow of weights of $M$.

We use the terminology which is defined in [17] as the cocycle conjugacy invariant as the terms to compute the flow of weights of $M \rtimes_{\alpha} G$.

We choose and fix a dominant weight $\phi$ on $M$, and identify $\left(P_{M}, F^{M}\right)$ with what was explained in 1.3. For each automorphism $\alpha$ in Aut $M$, the module $\bmod \alpha$ in Aut $P_{M}$ which commutes with $F^{M}$ is given by

$$
\bmod \alpha=\left.\operatorname{Ad} u \cdot \alpha\right|_{Z\left(M_{\phi}\right)},
$$

where $u$ is a unitary in $M$ satisfying $\phi \cdot \alpha^{-1}=\phi \cdot \operatorname{Ad} u$. If we set

$$
N(\alpha)=\alpha^{-1}\left(\left\{\operatorname{Ad} u \cdot \bar{\sigma}_{c}^{\phi} \mid u \in U(M), c \in Z_{\theta}^{1}\left(\mathbb{R}, U\left(Z\left(M_{\phi}\right)\right)\right)\right\}\right),
$$

$N(\alpha)$ is a normal subgroup of $G$. When $M$ is $A F D, N(\alpha)$ is the same as that in $[17]$ ([14; Theorem 1]). The characteristic invariant $\chi=[\lambda, \mu]$ in $\Lambda\left(G, N(\alpha), U\left(P_{M}\right)\right)$ and the modular invariant $\nu: N(\alpha) \rightarrow H_{F}^{1} M\left(\boldsymbol{R}, U\left(P_{M}\right)\right)$ are defined as follows: At first we choose unitaries $u_{h}$ in $M$ and unitary cocycles $c(h)$ in $Z_{\theta}^{1}\left(\boldsymbol{R}, U\left(Z\left(M_{\phi}\right)\right)\right)$ satisfying $\alpha_{h}=\operatorname{Ad} u_{h} \cdot \bar{\sigma}_{c(h)}^{\phi}, h \in N(\alpha)$. Identifying $\pi_{\theta}\left(M_{\phi}\right)$ with $M_{\phi}, \lambda$ and $\mu$ are defined by

$$
\begin{aligned}
u_{h} \bar{\sigma}_{c(h)}^{\phi}\left(u_{k}\right) & =\mu(h, k) \bar{\sigma}_{\partial \mu(h, k)}^{\phi}\left(u_{h k}\right) \\
& =u_{h k} \mu(h, k), \quad h, k \in N(\alpha),
\end{aligned}
$$

and

$$
\begin{aligned}
\alpha_{g}\left(u_{g-1 h g}\right)\left(D \phi \cdot \alpha_{g}^{-1}: D \phi\right)_{\left(\bmod \alpha_{g}\right)(c(g-1 h g))} & =\lambda(g, h) \bar{\sigma} \dot{\delta}_{\partial \lambda(g, h)}\left(u_{h}\right) \\
& =u_{h} \lambda(g, h), g \in G, h \in N(\alpha) .
\end{aligned}
$$

Here, for $u$ in $U\left(Z\left(M_{\phi}\right)\right)$, $\partial u$ means the coboundary defined by

$$
(\partial u)(s)=u * \theta_{s}(u), \quad s \in \boldsymbol{R} .
$$

And $\mu(h, k), h, k \in N(\alpha), \lambda(g, h), g \in G, h \in N(\alpha)$, and $c(h), h \in N(\alpha)$, satisfy the following relations :

$$
\begin{gathered}
c(h) c(k)=(\partial \mu(h, k)) c(h k), \\
\left(\bmod \alpha_{g}\right)\left(c\left(g^{-1} h g\right)\right)=(\partial \lambda(g, h)) c(h) .
\end{gathered}
$$

These two relations appear in [17; Theorem 5.14] somewhat mysteriously (at least to the author). They correspond exactly to commutativity of certain 
actions in our main result. And the modular invariant $\nu$ is defined by $\nu(h)=$ $[c(h)], h \in N(\alpha)$, which is a homomorphism.

Since we choose a weight $\phi$ on $M$, it is not clear that the above invariants are independent of the choice of $\phi$. But these depend only on the cocycle conjugacy class of $\alpha$. For details, see [17].

\subsection{Main Result}

We keep the above notations and state the main result in this article.

Theorem. Let $P_{M} \rtimes_{i d, \mu} N(\alpha)$ be the twisted crossed product of the abelian von Neumann algebra $P_{M}$ by $N(\alpha)$ with respect to the trivial action of $N(\alpha)$ and 2cocycle $\mu$. We consider the G-action $\gamma$ and $\boldsymbol{R}$-action $F$ on $P_{M} \rtimes_{i d, \mu} N(\alpha)$ defined by

$$
\begin{aligned}
& \gamma_{g}\left(\sum_{h \in N(\alpha)} c_{h} z_{h}\right)=\sum_{h \in N(\alpha)} \lambda(g, h)\left(\bmod \alpha_{g}\right)\left(c_{g-1 h g}\right) z_{h}, \quad g \in G, \\
& F_{t}\left(\sum_{h \in N(\alpha)} c_{h} z_{h}\right)=\sum_{h \in N(\alpha)} F_{t}^{M}\left(c_{h}\right) c(h, t) z_{h}, \quad t \in \boldsymbol{R} .
\end{aligned}
$$

Here, $z_{h}$ 's denote the generators coming from $N(\alpha)$, and $c(h, t)$ means the value of $c(h)$ at $t$.

Then the above two actions commute (hence $F$ induces the action on the fixed point subalgebra $\left.\left(P_{M} \rtimes_{i d, \mu} N(\alpha)\right)^{\gamma}\right)$, and $\left(\left(P_{M} \rtimes_{\imath d, \mu} N(\alpha)\right)^{\gamma},\left\{F_{t}\right\}_{t \in R}\right)$ is exactly the flow of weights of $M \rtimes_{\alpha} G$.

\section{§2. Canonical Extensions}

Let $\alpha: G \rightarrow$ Aut $M$ be an action of a locally compact group $G$ on a von Neumann algebra $M$. For a given faithful normal semi-finite weight $\phi$ on $M$ (with the dual weight $\tilde{\phi}$ on $\left.M \rtimes_{a} G\right)$ we can consider $\left(M \rtimes_{\alpha} G\right) \rtimes_{\sigma} \tilde{\phi} \mathbb{R}$. In the next proposition we will identify this algebra with a suitable crossed product relative to a certain $G$-action ([8; Proposition 12.1$]$ ). This result makes us possible to compute the flow of weights of $M \rtimes_{\alpha} G$ and is probably a folklore among specialists. However, the author fails to find a proof in the literature so that a proof will be presented.

Proposition 2.1. There exist an action $\tilde{\alpha}$ of $G$ on $M \rtimes_{\sigma} \phi \boldsymbol{R}$ and a spatial isomorphism $\Phi$ from $\left(M \rtimes_{\alpha} G\right) \rtimes_{\sigma} \tilde{\phi} \boldsymbol{R}$ onto $\left(M \rtimes_{\sigma} \dot{\boldsymbol{Q}} \boldsymbol{R}\right) \rtimes_{\tilde{a}} G$ such that

(i) For each $g \in G$, the automorphism $\tilde{\alpha}_{g}$ is characterized by

$$
\begin{aligned}
& \tilde{\alpha}_{g}\left(\pi_{\sigma} \phi(x)\right)=\pi_{\sigma \phi}\left(\alpha_{g}(x)\right), \quad x \in M, \\
& \tilde{\alpha}_{g}(\lambda(t))=\Delta_{G}(g)^{-i t} \pi_{\sigma} \phi\left(\left(D \phi \cdot \alpha_{g}{ }^{-1}: D \phi\right)_{t}\right) \lambda_{(}(t), \quad t \in \boldsymbol{R},
\end{aligned}
$$

where $\pi_{\sigma} \phi(x), \lambda(t)$, are the usual generators.

(ii) The isomorphism $\Phi$ is characterized by 


$$
\begin{aligned}
\Phi\left(\pi_{\sigma} \tilde{\phi}\left(\pi_{a}(x)\right)\right) & =\pi_{\tilde{\alpha}}\left(\pi_{\sigma} \phi(x)\right), \quad x \in M, \\
\Phi\left(\pi_{\sigma} \tilde{\phi}(\lambda(g))\right) & =\tilde{\lambda}(g), \quad g \in G, \\
\Phi(\Lambda(t)) & =\pi_{\tilde{a}}(\lambda(t)), \quad t \in \boldsymbol{R},
\end{aligned}
$$

where $\Lambda(t), t \in \boldsymbol{R}$, is the generator of $\left(M \rtimes_{a} G\right) \rtimes_{\sigma} \tilde{\phi} \boldsymbol{R}$ coming from $\boldsymbol{R}$ and $\tilde{\lambda}(g)$, $g \in G$, is that of $\left(M \rtimes_{\sigma} \phi \boldsymbol{R}\right) \rtimes_{\tilde{\alpha}} G$ coming from $G$.

(iii) $\Phi \cdot\left(\sigma_{t}^{\tilde{\delta}}\right)^{\wedge}=\left.\left(\left(\sigma_{t}^{\delta}\right)^{\wedge} \otimes 1\right)\right|_{\left(M \rtimes_{\sigma} \phi R\right) \rtimes_{\tilde{\alpha}} G} \cdot \Phi, \quad t \in \mathbb{R}$,

where $\left(\sigma^{\tilde{\phi}}\right)^{\wedge}$ is the dual action of $\sigma^{\tilde{\phi}}$ on $\left(M \rtimes_{a} G\right) \rtimes_{\sigma} \tilde{\phi} \boldsymbol{R},\left(\sigma^{\phi}\right)^{\wedge}$ is that of $\sigma^{\phi}$ on

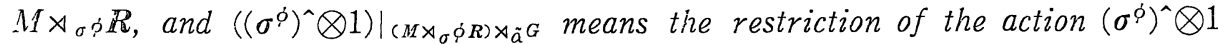
on $\left(M \rtimes_{\sigma} \phi \mathbb{R}\right) \otimes B\left(L^{2}(G)\right)$ to $\left(M \rtimes_{\sigma} \dot{\phi} \boldsymbol{R}\right) \rtimes_{\tilde{\alpha}} G$.

Proof. We may assume that $M$ acts standardly on the Hilbert space $H$.

We define the unitary operator $U$ on $L^{2}\left(\boldsymbol{R}, H \otimes L^{2}(G)\right)$ by

$$
(U \xi)(t)=\Delta_{\tilde{\phi}^{i t}}^{i} \xi(t), \quad \xi \in L^{2}\left(\mathbb{R}, H \otimes L^{2}(G)\right) .
$$

It is elementary to check

$$
\begin{aligned}
U \pi_{\sigma} \tilde{\phi}\left(\pi_{\alpha}(x)\right) U^{*} & =\pi_{a}(x) \otimes 1, & & x \in M, \\
U \pi_{\sigma} \tilde{\phi}(\lambda(g)) U^{*} & =\lambda(g) \otimes 1, & & g \in G, \\
U \Lambda(t) U^{*} & =\Delta_{\tilde{\phi}}^{i t} \otimes \lambda_{t}, & & t \in \boldsymbol{R},
\end{aligned}
$$

where $\lambda_{t}, t \in \mathbb{R}$, is the unitary operator on $L^{2}(\boldsymbol{R})$ defined by

$$
\left(\lambda_{t} f\right)(s)=f(s-t), \quad f \in L^{2}(\mathbb{R}), \quad s \in \mathbb{R} .
$$

Let $\left\{u_{g}\right\}_{g \in G}$ be the canonical implementation of $\alpha$. Let $V$ be the unitary operator on $L^{2}(G, H)$ defined by

$$
(V \xi)(g)=u_{g} \xi(g), \quad \xi \in L^{2}(G, H), \quad g \in G .
$$

We similarly have

$$
\begin{aligned}
& (V \otimes 1)\left(\pi_{\alpha}(x) \otimes 1\right)(V \otimes 1)^{*}=x \otimes 1 \otimes 1, \quad x \in M, \\
& (V \otimes 1)(\lambda(g) \otimes 1)(V \otimes 1)^{*}=u_{g} \otimes \lambda_{g} \otimes 1, \quad g \in G, \\
& (V \otimes 1)\left(\Delta_{\tilde{\phi}}^{i t} \otimes \lambda_{t}\right)(V \otimes 1)^{*}=V \Delta_{\tilde{\phi}}^{i t} V^{*} \otimes \lambda_{t}, \quad t \in \mathbb{R},
\end{aligned}
$$

where $\lambda_{g}, g \in G$, is the left regular representation of $G$ on $L^{2}(G)$. Further, we compute

$$
\left(V \Delta_{\tilde{\phi}^{i t}} V^{* \xi}\right)(g)=\Delta_{G}(g)^{i t} \Delta_{\phi, \delta} \cdot \alpha_{g^{-1}}{ }^{i t} \xi(g),
$$

Identifying $L^{2}(G) \otimes L^{2}(\boldsymbol{R})$ with $L^{2}(\boldsymbol{R}) \otimes L^{2}(G)$, we next define the unitary operator $W$ on $L^{2}(\boldsymbol{R} \times G, H)$ by 


$$
(W \xi)(t, g)=\Delta_{G}(g)^{-i t} \Delta_{\phi, \phi} \cdot \alpha_{g^{-1}}{ }^{-i t} \xi(t, g), \quad \xi \in L^{2}(\boldsymbol{R} \times G, H) .
$$

Then we have

$$
\begin{aligned}
W(x \otimes 1 \otimes 1) W^{*} & =\pi_{\sigma} \phi(x) \otimes 1, & & x \in M, \\
W\left(u_{g} \otimes \lambda_{g} \otimes 1\right) W^{*} & =\tilde{u}_{g} \otimes \lambda_{g}, & & g \in G, \\
W\left(V \Delta_{\tilde{\phi}}^{i t} V^{*} \otimes \lambda_{t}\right) W^{*} & =\lambda(t) \otimes 1, & & t \in \boldsymbol{R} .
\end{aligned}
$$

Here $\tilde{u}_{g}, g \in G$, is the unitary operator on $L^{2}(\boldsymbol{R}, H)$ defined by

$$
\left(\tilde{u}_{g} \eta\right)(t)=\Delta_{G}(g)^{-i t}\left(D \phi: D \phi \cdot \alpha_{g}{ }^{-1}\right)_{-t} u_{g} \eta(t), \quad \eta \in L^{2}(\boldsymbol{R}, H) .
$$

We remark that $\tilde{u}$ is a unitary representation of $G$ on $L^{2}(\boldsymbol{R}, H)$ and

$$
\begin{aligned}
& \tilde{u}_{g} \pi_{\sigma} \phi(x) \tilde{u}_{g}{ }^{*}=\pi_{\sigma \phi}\left(\alpha_{g}(x)\right), \quad x \in M, \\
& \tilde{u}_{g} \lambda(t) \tilde{u}_{g}{ }^{*}=\Delta_{G}(g)^{-\imath t} \pi_{\sigma} \phi\left(\left(D \phi \cdot \alpha_{g}{ }^{-1}: D \phi\right)_{t}\right) \lambda(t), \quad t \in \boldsymbol{R} .
\end{aligned}
$$

Hence we get an action $\tilde{\alpha}(=\operatorname{Ad} \tilde{u})$ of $G$ on $M \rtimes_{\sigma} \phi \boldsymbol{R}$.

Finally, if we define the unitary operator $X$ on $L^{2}\left(G, H \otimes L^{2}(\boldsymbol{R})\right)$ by

$$
(X \xi)(g)=\tilde{u}_{g} * \xi(g), \quad \xi \in L^{2}\left(G, H \otimes L^{2}(\boldsymbol{R})\right),
$$

we obtain an isomorphism $\Phi:\left(M \rtimes_{\alpha} G\right) \rtimes_{\sigma} \tilde{\phi} \boldsymbol{R} \rightarrow\left(M \rtimes_{\sigma} \phi \boldsymbol{R}\right) \rtimes_{\tilde{\alpha}} G$ with the desired properties (i) and (ii).

From the definition of the dual action it is easy to see that the above $\Phi$ satisfies the property (iii).

q.e.d.

Notice that the automorphism $\tilde{\alpha}_{g}$ in the above proof is exactly the canonical extension of $\alpha_{g}$ in the sense of Haagerup-Størmer, [8].

Remark 2.2. If $\phi$ is another faithful normal semi-finite weight on $M$, there exists an action $\tilde{\alpha}^{\psi}$ of $G$ on $M \rtimes_{\sigma} \psi \boldsymbol{R}$. But it is easy to check that $\tilde{\alpha}$ and $\tilde{\alpha}^{\dot{\psi}}$ are conjugate via the usual isomorphism from $M \rtimes_{\sigma} \phi \boldsymbol{R}$ onto $M \rtimes_{\sigma} \dot{ } \boldsymbol{R}$ constructed from Connes' Radon-Nikodym cocycle ([18; Proposition 3.5]).

From now on let us assume that $M$ is a factor and $G$ is discrete, and we set $\tilde{M}=M \rtimes_{\sigma} \dot{R} \boldsymbol{R}, \theta$ the dual action of $\sigma^{\phi}$. Using the action $\tilde{\alpha}$ in Proposition 2.1 , we set $N(\tilde{\alpha})=\tilde{\alpha}^{-1}(\operatorname{Int} \tilde{M})$. Then the characteristic invariant $\tilde{\chi}=[\tilde{\lambda}, \tilde{\mu}]$ in $\Lambda(G, N(\tilde{\alpha}), U(Z(\tilde{M})))$ is defined as follows: At first we choose unitary operators $\tilde{u}_{h}$ in $\tilde{M}$ satisfying $\tilde{\alpha}_{h}=\operatorname{Ad} \tilde{u}_{h}, h \in N(\tilde{\alpha})$. Then $\tilde{\lambda}$ and $\tilde{\mu}$ are defined by

$$
\begin{aligned}
& \tilde{u}_{h} \tilde{u}_{k}=\tilde{\mu}(h, k) \tilde{u}_{h k}, \quad h, k \in N(\tilde{\alpha}), \\
& \tilde{\alpha}_{g}\left(\tilde{u}_{g^{-1} h g}\right)=\tilde{\lambda}(g, h) \tilde{u}_{h}, \quad g \in G, \quad h \in N(\tilde{\alpha}) .
\end{aligned}
$$

For details, we refer to $[10],[11],[16]$. 
Lemma 2.3. With the same notations as above,

(i) $\theta_{t} \cdot \tilde{\alpha}_{g}=\tilde{\alpha}_{g} \cdot \theta_{t}, t \in \boldsymbol{R}, g \in G$,

(ii) For $g \notin N(\tilde{\alpha}), \tilde{\alpha}_{g}$ is free in the sense of [13].

Proof. (i) It is sufficient to check the equality against generators, and details are left to the reader ([8; Proposition 12.2]).

(ii) Let $p(g)$ be the inner part of $\tilde{\alpha}_{g}$, that is, $p(g)$ is the largest central projection with the following properties:

$$
\begin{aligned}
& \tilde{\alpha}_{g}(p(g))=p(g), \\
& \left.\tilde{\alpha}_{g}\right|_{\tilde{M} p(g)} \text { is inner. }
\end{aligned}
$$

From (i),

$$
\theta_{t}(p(g))=p(g), \quad t \in \boldsymbol{R} .
$$

Hence we have, by the ergodicity of $\theta$,

$$
p(g)=0 \text { or } 1 \text {. }
$$

q.e.d.

Lemma 2.4. Let $Z(\tilde{M}) \rtimes_{\imath d, \tilde{\mu}} N(\tilde{\alpha})$ be the twisted crossed product of $Z(\tilde{M})$ by $N(\tilde{\alpha})$ with respect to the trivial action of $N(\tilde{\alpha})$ and the 2-cocycle $\tilde{\mu}$.

The map II from $Z(\tilde{M}) \rtimes_{i a, \tilde{\mu}} N(\tilde{\alpha})$ into $\tilde{M} \rtimes_{\tilde{\alpha}} G$ defined by

$$
\Pi\left(\sum_{h \in N(\tilde{\alpha})} c_{h} z_{h}\right)=\sum_{h \in N(\tilde{\alpha})} \pi_{\tilde{\alpha}}\left(c_{h}\right) \pi_{\tilde{\alpha}}\left(\tilde{u}_{h}\right) \tilde{\lambda}(h)^{*}, \quad c_{h} \in Z(\tilde{M}),
$$

is a *anti-isomorphism onto $\tilde{M}^{\prime} \cap\left(\tilde{M} \rtimes_{\tilde{\alpha}} G\right)$ and satisfies

$$
\Pi\left(\left(Z(\tilde{M}) \rtimes_{i d, \tilde{\mu}} N(\tilde{\alpha})\right)^{\gamma}\right)=Z\left(\tilde{M} \rtimes_{\tilde{\alpha}} G\right) .
$$

Here $\gamma$ is the action of $G$ on $Z(\tilde{M}) \rtimes_{\imath a, \tilde{\mu}} N(\tilde{\alpha})$ defined by

$$
\gamma_{g}\left(\sum_{h \in N(\tilde{\alpha})} c_{h} z_{h}\right)=\sum_{h \in N(\tilde{\alpha})} \tilde{\lambda}(g, h) \tilde{\alpha}_{g}\left(c_{g-1 h g}\right) z_{h},
$$

and $z_{h}, h \in N(\tilde{\alpha})$, is the generator of $Z(\tilde{M}) \rtimes_{i d, \tilde{\mu}} N(\tilde{\alpha})$ coming from $N(\tilde{\alpha})$.

Proof. Thanks to Lemma 2.3, a proof is the same as that in [10] or [11]. Thus we give here only a sketch.

We identify $\pi_{\tilde{\alpha}}(\tilde{M})$ with $\tilde{M}$. Let $X=\sum_{g \in G} \tilde{x}_{g} \tilde{\lambda}(g)$ be an element in $\tilde{M} \rtimes_{\tilde{a}} G$. If $X$ is in $\tilde{M}^{\prime} \cap\left(\tilde{M} \rtimes_{\tilde{\alpha}} G\right)$, we have

$$
\sum_{g \in G} \tilde{x} \tilde{x}_{g} \tilde{\lambda}(g)=\sum_{g \in G} \tilde{x}_{g} \tilde{\alpha}_{g}(x) \tilde{\lambda}(g), \quad \tilde{x} \in \tilde{M} .
$$

Hence $X$ is of the form $\sum_{h \in N(\tilde{\alpha})} c_{h} \tilde{u}_{h} \tilde{\lambda}(h)^{*}$ for some $c_{h} \in Z(\tilde{M})$. Moreover, if $X$ is in $Z\left(\tilde{M} \rtimes_{\hat{\alpha}} G\right), X$ must commute with $\tilde{\lambda}(g), g \in G$. We compute 


$$
\begin{aligned}
\sum_{h \in N(\tilde{\alpha})} c_{h} \tilde{u}_{h} \tilde{\lambda}(h)^{*} & =\tilde{\lambda}(g)\left(\sum_{h \in N(\tilde{\alpha})} c_{h} u_{h} \tilde{\lambda}(h)^{*}\right) \tilde{\lambda}(g)^{*} \\
& =\sum_{h \in N(\tilde{\alpha})} \tilde{\alpha}_{g}\left(c_{h}\right) \tilde{\alpha}_{g}\left(\tilde{u}_{h}\right) \tilde{\lambda}\left(g h g^{-1}\right)^{*} \\
& =\sum_{h \in N(\tilde{\alpha})} \tilde{\alpha}_{g}\left(c_{g-1 h g}\right) \tilde{\alpha}_{g}\left(\tilde{u}_{g-1} g\right) \tilde{\lambda}(h)^{*} \\
& =\sum_{h \in N(\tilde{\alpha})} \tilde{\lambda}(g, h) \tilde{\alpha}_{g}\left(c_{g-1 h g}\right) \tilde{u}_{h} \tilde{\lambda}(h)^{*}
\end{aligned}
$$

q.e.d.

\section{$\S 3$. Computation of the Flow of Weights}

We will keep the previous notations, and from now on let $M$ be a type III factor.

We note that our main interest is not the algebra $M \rtimes_{\alpha} G$ itself, but the isomorphism class of $M \rtimes_{\alpha} G$. Therefore, we may choose another action $\beta$ of $G$ on $M$ cocycle conjugate to $\alpha$. More precisely, if two actions $\alpha$ and $\beta$ of $G$ on $M$ are cocycle conjugate, $M \rtimes_{\alpha} G$ and $M \rtimes_{\beta} G$ are isomorphic and hence their flows are conjugate ([18; Proposition 3.5, Corollary 3.6 and Proposition 4.2]). By [17; Lemma 5.11], we may and do assume the following:

( I ) $\dot{\phi}$ is a dominant weight on $M$ such that

$$
\phi \cdot \alpha_{g}=\phi, \quad g \in G,
$$

(II) For the continuous decomposition $M=M_{\phi} \rtimes_{\theta} \boldsymbol{R}$,

$$
\begin{gathered}
\left(\alpha_{0}\right)_{g} \cdot \theta_{s}=\theta_{s} \cdot\left(\alpha_{0}\right)_{g}, \quad g \in G, \quad s \in \boldsymbol{R}, \\
\left\{\begin{array}{l}
\alpha_{g}\left(\pi_{\theta}(x)\right)=\pi_{\theta}\left(\left(\alpha_{0}\right)_{g}(x)\right), \quad x \in M_{\phi}, \\
\alpha_{g}(\lambda(s))=\lambda(s), \quad s \in \boldsymbol{R},
\end{array}\right.
\end{gathered}
$$

where $\left(\alpha_{0}\right)_{g}$ is the automorphism on $M_{\dot{\phi}}$ induced by $\alpha_{g}$.

Lemma 3.1. There exists an isomorphism $\Psi$ from $\tilde{M}$ onto $M_{\phi} \otimes B\left(L^{2}(\boldsymbol{R})\right)$ such that

$$
\begin{aligned}
& \Psi \cdot \tilde{\alpha}_{g}=\left(\left(\alpha_{0}\right)_{g} \otimes 1\right) \cdot \Psi, \quad g \in G, \\
& \Psi \cdot\left(\boldsymbol{\sigma}_{t}^{\phi}\right)^{\wedge}=\left(\theta_{t} \otimes \operatorname{Ad} \rho_{t}\right) \cdot \Psi, \quad t \in \boldsymbol{R},
\end{aligned}
$$

Proof. Since $\sigma^{\dot{\phi}}$ is the dual action of $\theta$, Takesaki duality shows that there exists an isomorphism $\Psi: \tilde{M} \rightarrow M_{\phi} \otimes B\left(L^{2}(\boldsymbol{R})\right)$ such that

and

$$
\begin{array}{rlrl}
\Psi\left(\pi_{\sigma} \phi\left(\pi_{\theta}(x)\right)\right)=\pi_{\theta}(x), & & x \in M_{\phi}, \\
\Psi\left(\pi_{\sigma} \phi(\lambda(t))\right) & =1 \otimes \lambda_{t}, & & t \in \boldsymbol{R}, \\
\Psi(\lambda(s))=1 \otimes v_{s}, & & s \otimes R,
\end{array}
$$

$$
\Psi \cdot\left(\sigma_{t}^{\phi}\right)^{\wedge}=\left(\theta_{t} \otimes \operatorname{Ad} \rho_{t}\right) \cdot \Psi, \quad t \in \boldsymbol{R} .
$$


By the above assumptions, this $\Psi$ satisfies the other required property. q.e.d.

Lemma 3.2. There exists an isomorphism $\Psi_{0}$ from $Z(\tilde{M})$ onto $Z\left(M_{\phi}\right)$ such that

$$
\begin{array}{ll}
\left.\Psi_{0} \cdot \tilde{\alpha}_{g}\right|_{Z(\widetilde{M})}=\left.\left(\alpha_{0}\right)_{g}\right|_{Z\left(M_{\phi}\right)} \cdot \Psi_{0}, & g \in G, \\
\left.\Psi_{0} \cdot\left(\sigma_{t}^{\phi}\right)^{\wedge}\right|_{Z(\widetilde{M})}=\left.\theta_{t}\right|_{Z\left(M_{\phi}\right)} \cdot \Psi_{0}, & t \in \boldsymbol{R} .
\end{array}
$$

Proof. If we define the isomorphism $\Omega: Z\left(M_{\phi}\right) \rightarrow Z(\tilde{M})$ by

$$
\Omega(x)=\Psi^{-1}(x \otimes 1), \quad x \in Z\left(M_{\phi}\right),
$$

then $\Psi_{0}=\Omega^{-1}$ has the desired properties.

q.e.d.

In our set-up, for each $g \in G$, the $\operatorname{module} \bmod \alpha_{g}$ is given by

$$
\bmod \alpha_{g}=\left.\left(\alpha_{0}\right)_{g}\right|_{z\left(M_{\phi}\right)}, \quad g \in G .
$$

And for $\alpha_{h}=\operatorname{Ad} u_{h} \cdot \bar{\sigma}_{c(h)}^{\phi}, h \in N(\alpha)$, the assumption (I) shows that each unitary $u_{h}$ must belong to $M_{\phi}$. This means that each $\alpha_{h}, h \in N(\alpha)$, is of the form Ad $\pi_{\theta}\left(u_{h}\right) \cdot \bar{\sigma}_{c(h)}^{\dot{\phi}}$. Further, $\lambda$ and $\mu$ are defined by the following relations:

$$
\begin{array}{ll}
\pi_{\theta}\left(u_{h}\right) \pi_{\theta}\left(u_{k}\right)=\pi_{\theta}(\mu(h, k)) \pi_{\theta}\left(u_{h_{k}}\right), & h, k \in N(\alpha), \\
\alpha_{g}\left(\pi_{\theta}\left(u_{g-1 h g}\right)\right)=\pi_{\theta}(\lambda(g, h)) \pi_{\theta}\left(u_{h}\right), & g \in G, \quad h \in N(\alpha) .
\end{array}
$$

By the assumption (II.2), we have

$$
c(h, t)=u_{h} * \theta_{t}\left(u_{h}\right), \quad t \in \boldsymbol{R},
$$

because we compute

$$
\begin{aligned}
\lambda(t) & =\alpha_{h}(\lambda(t)) \\
& =\pi_{\theta}\left(u_{h}\right) \pi_{\theta}(c(h, t)) \lambda(t) \pi_{\theta}\left(u_{h}{ }^{*}\right) \\
& =\pi_{\theta}\left(u_{h} c(h, t) \theta_{t}\left(u_{h}\right)^{*}\right) \lambda(t) .
\end{aligned}
$$

Though the following lemma is implicit in the proof of [9; Proposition 5.4], we give a proof for the sake of completeness.

Lemma 3.3. Let $N\left(\alpha_{0}\right)=\left(\alpha_{0}\right)^{-1}\left(\operatorname{Int} M_{\dot{\rho}}\right)$, and $\chi_{0}=\left[\lambda_{0}, \mu_{0}\right]$ be the characteristic invariant in $\Lambda\left(G, N\left(\alpha_{0}\right), U\left(Z\left(M_{\dot{\varphi}}\right)\right)\right)$.

(i) $N(\alpha)=N\left(\alpha_{0}\right)$,

(ii) $\chi=\chi_{0}$ in $\Lambda\left(G, N(\alpha), U\left(Z\left(M_{\phi}\right)\right)\right)$.

Proof. (i) If $u \in N(\alpha)$, there exist a unitary $u_{h}$ in $M_{\phi}$ and a cocycle $c(h)$ in $Z_{\theta}^{1}\left(\boldsymbol{R}, U\left(Z\left(M_{\phi}\right)\right)\right)$ such that $\alpha_{h}=\operatorname{Ad} \pi_{\theta}\left(u_{h}\right) \cdot \overline{\boldsymbol{\sigma}}_{c(h)}^{\phi}$. From the definition of $\alpha_{0}$ and the property of the extended modular automorphism it follows that $\left(\alpha_{0}\right)_{h}=$ Ad $u_{h}$. Hence we have $N(\alpha) \subseteq N\left(\alpha_{0}\right)$. Conversely, if $h \cong N\left(\alpha_{0}\right),\left(\alpha_{0}\right)_{h}=\operatorname{Ad} u_{h}$ for 
some $u_{h} \in U\left(M_{\phi}\right)$, that is, Ad $\pi_{\theta}\left(u_{h}{ }^{*}\right) \cdot \alpha_{h}=1$ on $\pi_{\theta}\left(M_{\phi}\right)$. So, by [3; IV. Theorem 2.2], $\alpha_{h}$ must be of the form $\operatorname{Ad} \pi_{\theta}\left(u_{h}\right) \cdot \bar{\sigma}_{c(h)}^{\phi}$.

(ii) From the above argument and the definition of the characteristic invariant, we have $\chi=\chi_{0}$.

q.e.d.

Proof of the main theorem. It is sufficient to compute the pair $\left(Z\left(M \rtimes_{\alpha} G\right.\right.$ $\left.\left.\rtimes_{\sigma \tilde{\phi}} \boldsymbol{R}\right),\left(\sigma^{\tilde{\phi}}\right)^{\wedge}\right)$. We may identify $\left(P_{M}, F^{M}\right)$ with $\left(Z\left(M_{\dot{\phi}}\right), \theta\right)$.

From the condition (1.4.1), we compute

$$
\begin{aligned}
F_{t}\left(z_{h}\right) F_{t}\left(z_{k}\right) & =c(h, t) z_{h} c(k, t) z_{k} \\
& =c(h, t) c(k, t) \mu(h, k) z_{h k} \\
& =\theta_{t}(\mu(h, k)) c(h k, t) z_{h k} \\
& =F_{t}\left(\mu(h, k) z_{h k}\right) \\
& =F_{t}\left(z_{h} z_{k}\right) .
\end{aligned}
$$

We also compute

$$
\begin{aligned}
F_{s} \cdot F_{t}\left(c_{h} z_{h}\right) & =F_{s}\left(\theta_{t}\left(c_{h}\right) c(h, t) z_{h}\right) \\
& =\theta_{s+t}\left(c_{h}\right) \theta_{s}(c(h, t)) c(h, s) z_{h} \\
& =\theta_{s+t}\left(c_{h}\right) c(h, s+t) z_{h} \\
& =F_{s+t}\left(c_{h} z_{h}\right) .
\end{aligned}
$$

Hence we conclude that $F$ is an action of $\boldsymbol{R}$ on $Z\left(M_{\phi}\right) \rtimes_{i d, \mu} N(\alpha)$. And, by (1.4.2), we compute

$$
\begin{aligned}
F_{t} \cdot \gamma_{g}\left(c_{h} z_{h}\right) & =F_{t}\left(\lambda(g, h)\left(\bmod \alpha_{g}\right)\left(c_{g-1} g\right) z_{h}\right) \\
& =\theta_{t}(\lambda(g, h)) \theta_{t}\left(\left(\bmod \alpha_{g}\right)\left(c_{g-1 h g}\right)\right) c(h, t) z_{h} \\
& =\lambda(g, h)\left(\bmod \alpha_{g}\right)\left(\theta_{t}\left(c_{g-1 h g}\right)\right)\left(\bmod \alpha_{g}\right)\left(c\left(g^{-1} h g, t\right)\right) z_{h} \\
& =\gamma_{g}\left(\theta_{t}\left(c_{h}\right) c(h, t) z_{h}\right) \\
& =\gamma_{g} \cdot F_{t}\left(c_{h} z_{h}\right) .
\end{aligned}
$$

Hence it follows that $F$ induces an action on $\left(Z\left(M_{\phi}\right) \rtimes_{i d, \mu} N(\alpha)\right)^{\gamma}$.

From the results in $\S 2$ and $\S 3, Z\left(M \rtimes_{\alpha} G \rtimes_{\sigma} \tilde{\phi} \boldsymbol{R}\right)$ is isomorphic to $\left(Z\left(M_{\phi}\right)\right.$ $\left.\rtimes_{i d, \mu} N(\alpha)\right)^{\gamma}$, and if we note the definition of $\Pi$ as in Lemma $2.4,\left(\sigma^{\tilde{\phi}}\right)^{\wedge}$ is conjugate to $F$ via the above isomorphism. 


\section{References}

[1] Connes, A., Une classification des facteurs de type III, Ann. Sci. Écol. Norm. Sup., 6 (1973), 133-252.

[2] - Classification of injective factors, Ann. Math., 104 (1976), 73-116.

[3] Connes, A. and Takesaki, M., The flow of weights on a factor of type III, Tohoku Math. J., 29 (1977), 473-575.

[4] van Daele, A., Continuous crossed products and type III von Neumann algebras, London Math. Lecture Notes Series 31, 1978.

[5] Haagerup, U., The standard form of von Neumann algebras, Math. Scand, 37 (1975), 271-283.

[6] - On the dual weights for crossed products of von Neumann algebras. I, Math. Scand., 43 (1978), 99-118.

[7] - Connes' bicentralizer problem and uniqueness of the injective factor of type $\mathrm{III}_{1}$, Acta Math., 158 (1987), 95-147.

[8] Haagerup, U. and Størmer, E., Equivalence of normal states on von Neumann algebras and the flow of weights, Advance in Math., to appear.

[9] - Pointwise inner automorphisms of von Neumann algebras, preprint.

[10] Jones, V., Actions of finite groups on the hyperfinite type $\mathrm{II}_{1}$ factor, Mem. Amer. Math. Soc., 237 (1980).

[11] Jones, V. and Takesaki, M., Actions of compact abelian groups on semi-finite injective factors, Acta Math., 153 (1984), 213-258.

[12] Kadison, R. and Ringrose, J., Fundamentals of the theory of operator algebras, vol. II, Academic Press, 1986.

[13] Kallman, R., A generalization of free action, Duke Math. J., 36 (1969), 781-789.

[14] Kawahigashi, Y., Sutherland, C. and Takesaki, M., The structure of the automorphism group of an injective factor and the cocycle conjugacy of discrete abelian group actions, preprint.

[15] Krieger, W., On ergodic flows and isomorphism of factors, Math. Ann., 223 (1976), 19-70.

[16] Ocneanu, A., Actions of discrete amenable groups on von Neumann algebras, Springer Lecture Notes in Math., 1138 (1985).

[17] Sutherland, C. and Takesaki, M., Actions of discrete amenable groups on injective factors of type III $\lambda, \lambda \neq 1$, Pacific J. Math., 137 (1989), 405-444.

[18] Takesaki, M., Duality for crossed products and the structure of von Neumann algebras of type III, Acta Math., 131 (1973), 249-310. 